\title{
anatomie musculaire
}

\section{du sourire}

\author{
«La pensée est au mot ce que le sentiment \\ est à l'expression faciale» \\ Charles Bell
}

RÉSUMÉ Les déplacements cutanés caractéristiques du sourire sont liés à l'action des muscles faciaux organisés en sangles labiale et orbito-palpébrales. Si l'on décrit classiquement l'action de ces muscles de façon isolée, il faut garder à l'esprit que l'importante variabilité inter- et intra-individuelle des expressions faciales, et notamment du sourire, repose sur l'action combinée de ces éléments musculaires au sein de structures spécifiques: SMAS (Système MusculoAponévrotique Superficiel), sillon naso-labial et modiolus. L'interaction de ces structures confère aux régions labiales

\section{G. CLÈDES}

AHU,

Département d'Anatomie

Cervico-Céphalique,

Faculté de chirurgie dentaire,

Université Paris VII Denis-Diderot.

\section{R. FELIZARDO}

Ancien AHU, chargé de cours

Département d'Anatomie

Cervico-Céphalique,

Faculté de chirurgie dentaire,

Université Paris VII Denis-Diderot.

\section{P. CARPENTIER}

PU-PH,

Département d'Anatomie

Cervico-Céphalique,

Faculté de chirurgie dentaire,

Université Paris VII Denis-Diderot. et péri-labiales une architecture musculo-fibreuse tridimensionnelle spécialisée.

\section{MOTS CLÉS}

sourire

anatomie musculaire

région labiale

SMAS

sillon naso-labial

modiolus 


\section{introduction}

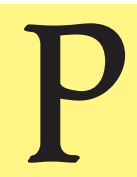

armi les moyens non verbaux de communication propres à l'Homme, le sourire est une expression typique dont les déplacements cutanés sont connus, et reconnus de tous : élargissement de la fente orale et de la base du nez, élévation des commissures labiales et de la lèvre supérieure, et plissement des yeux. Mais connaissons-nous réellement le substrat ana- tomique de cet outil fondamental de nos relations sociales ? Pour tenter de répondre à cette question, nous aborderons dans un premier temps de manière systématique les différents éléments musculaires intervenant dans le sourire, avant d'étudier les interrelations complexes existant entre eux, conférant aux régions labiale et périlabiale une architecture fonctionnelle spécialisée.

\section{sangles musculaires intervenant dans le sourire}

(fig. 1)

Le sourire est caractérisé par une ascension des tissus faciaux associée à une dilatation des orifices transversaux[1,2], résultant de l'action conjuguée de nombreux muscles faciaux, schématiquement organisés en deux sangles[3] :

- une sangle principale, impaire et médiane : la sangle buccale ;

- deux sangles complémentaires, paires et symétriques : les sangles orbitopalpébrales.

Rappelons que les muscles faciaux, dérivés du deuxième arc branchial, reçoivent leur innervation motrice $d u$ nerf facial (VII), et possèdent une insertion cutanée responsable de leur fonction de mimique.

\section{la sangle buccale}

La sangle buccale consiste en une couronne musculaire organisée de manière radiée autour $\mathrm{du}$ muscle orbiculaire de la bouche, contrariant son action sphinctérienne.

Ce muscle, situé dans l'épaisseur des lèvres, sans insertion osseuse directe, est constitué de deux parties distinctes : une couche profonde composée de fibres semi-circulaires en continuité avec les muscles buccinateurs en arrière, et une couche superficielle formée par l'enchevêtrement des muscles peauciers commissuraux. Pour Couly, ces deux plans musculaires assument des rôles complémentaires, conférant à l'orifice oral une double fonctionnalité de «sphincter digestif à fonction mimique intégrée»[4].

\section{MUSCLES DILATATEURS DE LA FENTE ORALE}

\section{Muscle buccinateur}

Le buccinateur est un muscle plat, s'insérant en arrière sur le maxillaire, la mandibule et le ligament ptérygo-man- 


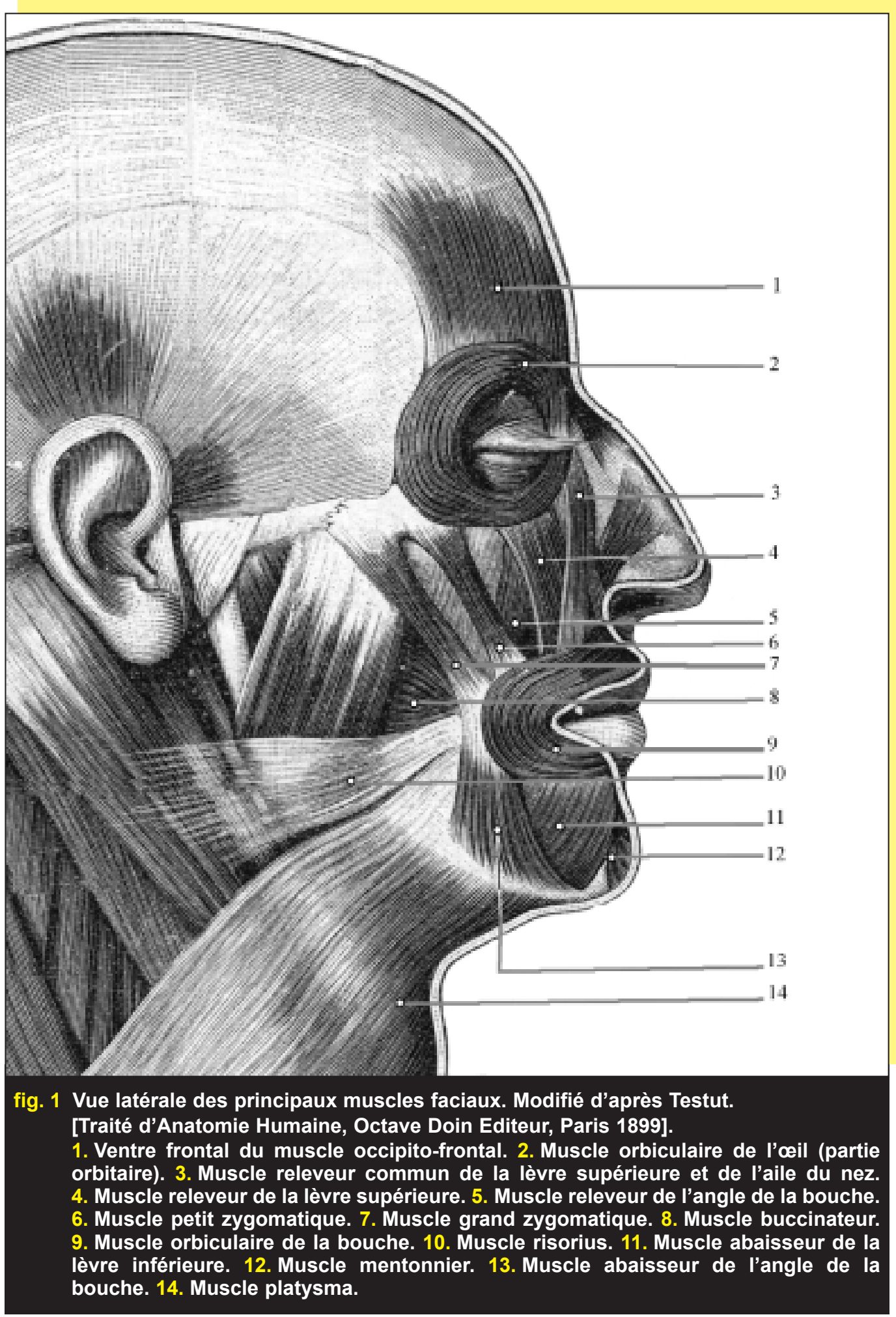


dibulaire. Ses fibres, essentiellement longitudinales, se terminent au niveau des commissures labiales en s'entrecroisant. Peu expressif, le buccinateur crée, en tractant les commissures labiales vers l'arrière, un sourire de type viscéral, reflétant pour certains la satiété et la satisfaction[2], pour d'autres l'ironie[3].

\section{Muscle risorius}

Le risorius est un muscle inconstant, tendu du fascia masséterin à la commissure labiale. Il intervient dans le sourire comme auxiliaire du muscle buccinateur, amenant les commissures en arrière et en dehors. Selon certains auteurs, sa contraction fait naître la fossette $d u$ sourire[3]. Pour d'autres, son apparition serait liée à un écart particulièrement important entre les insertions des muscles risorius et grand zygomatique[5], ou à une insertion cutanée $d u$ chef inférieur $d u$ grand zygomatique lorsque celui-ci est bifide[6].

\section{MUSCLES ÉLÉVATEURS DE LA COMMISSURE}

\section{Muscle grand zygomatique}

Le muscle grand zygomatique s'étend de l'os zygomatique à la commissure labiale qu'il porte en haut et en dehors. C'est le muscle de la joie de Duchenne de Boulogne[7], dont la contraction produit d'importantes modifications faciales : au-delà de l'ascension de la commissure, elle accuse le galbe de la pommette en refoulant les parties molles de la joue, élève la paupière inférieure, et crée un plissement cutané latéro-orbitaire caractéristique («rides de la patte d'oie») ${ }^{[1,3]}$. Cette dernière fonction s'explique par les connexions étroites existant entre les muscles grand zygomatique et orbiculaire de l'œil au niveau du canthus externe, et en dehors de lui[8].

\section{Muscle releveur de l'angle de la bouche}

Le muscle releveur de l'angle de la bouche est un muscle quadrilatère s'étendant de la fosse canine à la commissure, qu'il dépasse pour se terminer à la face profonde de la peau de la lèvre inférieure. Il élève donc la commissure et la lèvre inférieure.

Au cours du sourire, la commissure est la structure faciale qui subit le déplacement quantitativement le plus important, puisqu'il est en moyenne de $14 \mathrm{~mm}$. L'action combinée des muscles grand zygomatique et releveur de l'angle l'amène en haut et en dehors selon une direction moyenne de 40 degrés par rapport à l'horizontale[9]. Selon Rubin, la plupart des sujets (67\% de son échantillon) présente, au cours du sourire, un déplacement prépondérant des commissures, réalisant ce qu'il nomme un sourire «Mona Lisa»[10].

\section{MUSCLES ÉLÉVATEURS DE LA LÈVRE SUPÉRIEURE}

\section{Muscle releveur de la lèvre supérieure}

Le muscle releveur de la lèvre supérieure, légèrement oblique en bas et en dedans, s'étend du rebord inférieur de l'orbite à la face profonde de la lèvre supérieure qu'il élève. De forme triangulaire à sommet inférieur, ses fibres recouvrent le pédicule infra-orbitaire. 


\section{Muscle releveur commun de la lèvre supérieure et de l'aile du nez}

Le muscle releveur commun de la lèvre supérieure et de l'aile du nez s'étend du rebord médial de l'orbite à la peau du bord postérieur de l'aile du nez et de la lèvre supérieure. Légèrement oblique en bas et en dehors, il élève l'aile du nez et la lèvre supérieure.

\section{Muscle petit zygomatique}

Le muscle petit zygomatique, inconstant, s'insère sur l'os zygomatique en avant du muscle grand zygomatique. Le plus souvent, il rejoint le muscle releveur de la lèvre supérieure, mais peut aussi s'insérer directement à la peau de la lèvre supérieure, qu'il attire alors en haut et en dehors. Il agit dans le sourire en synergie avec les muscles grand zygomatique et releveur de la lèvre supérieure.

Au cours du sourire, la portion médiane de la lèvre supérieure s'élève quasi verticalement, sur une distance moyenne de $6 \mathrm{~mm}$. Ses portions latérales subissent quant à elles un mouvement vers le haut et le dehors d'une direction moyenne de 43 degrés par rapport à l'horizontale, et d'une amplitude moyenne de $11 \mathrm{~mm}$. L'amplitude de mouvement de la lèvre supérieure est donc moins importante que celle de la commissure labiale, mais s'effectue selon une direction plus verticale, liée à la faible obliquité des muscles élévateurs de la lèvre supérieure[9]. Pour Rubin, l'élévation de la lèvre supérieure prédomine néanmoins chez $31 \%$ des sujets, réalisant un sourire canin, ou carnassier, caractérisé par une exposition importante des canines maxillaires[10]. La partie latérale de l'aile du nez s'élève de manière plus verticale que la lèvre supérieure (48 degrés par rapport à l'horizontale), liée à l'orientation du muscle releveur commun de la lèvre supérieure et de l'aile du nez, avec une amplitude de déplacement moindre (7 mm en moyenne) [9]. Selon Rouvière, le déplacement de l'aile du nez serait aussi lié à l'action du muscle dilatateur de la narine[1].

\section{MUSCLE ABAISSEUR DE LA LÈVRE INFÉRIEURE}

Le muscle abaisseur de la lèvre inférieure s'étend du tiers antérieur du bord basilaire de la mandibule à la peau de la lèvre inférieure, la partie supérieure du bord antérieur des muscles droit et gauche s'unissant sur la ligne médiane. $\mathrm{Sa}$ contraction amène en bas et en dehors l'hémi-lèvre inférieure correspondante.

Bien que le sourire soit principalement caractérisé par des mouvements d'élévation, il fait classiquement intervenir un abaissement de la lèvre inférieure. La portion médiane de la lèvre inférieure s'abaisse ainsi verticalement sur une distance moyenne de $3 \mathrm{~mm}$. Ses portions latérales effectuent quant à elles un mouvement en dehors d'une amplitude moyenne de $7 \mathrm{~mm}$, globalement horizontal[9]. Ces mouvements sont notamment marqués chez les sujets présentant, selon Rubin, un sourire "carré», découvrant largement les dents maxillaires et mandibulaires, par l'action combinée de tous les muscles de la sangle labiale[10]. Les mouvements de la lèvre inférieure présentent néanmoins une plus grande variabilité interindividuelle que ceux de la lèvre supérieure, pouvant conduire, chez certains sujets, à une élévation et non un abaissement de celle-ci[9]. 
MUSCLES ABAISSEURS DE LA COMMISSURE

La participation au sourire des muscles abaisseurs de la commissure labiale (muscles abaisseurs de l'angle de la bouche et platysma) intervient essentiellement chez ces mêmes sujets à sourire "carré», qui ne représentent qu'une faible part de la population [2\% pour Rubin[10], 1 sujet sur 36 pour Paletz[9]].

\section{- sangle orbito-palpébrale}

L'action de la sangle orbito-palpébrale apporte toute sa spontanéité au sourire : sa contraction est indispensable à l'obtention d'un rire «vrai», comme l'a qualifié en son temps Duchenne de Boulogne[7]. Les psychologues lui rendent aujourd'hui hommage en opposant le «Duchenne smile», caractérisé par une activité musculaire péri-orbitaire, traduisant un ressenti émotionnel de plaisir, au «Non-Duchenne smile», sourire volontaire à vocation sociale[11] Cette sangle est essentiellement constituée du muscle orbiculaire de l'œil qui, par sa portion orbitaire disposée concentriquement autour de l'orifice palpébral, et sa portion palpébrale armant les paupières, agit en synergie avec les muscles zygomatiques au cours du sourire, aboutissant à un plissement des tissus cutanés latéro-orbitaires («rides de la patte d'oie») et un rétrécissement de la fente palpébrale. S'y ajoute l'action du muscle occipito-frontal qui peut, selon la séquence de contraction de ses portions frontale et occipitale, élever les sourcils et la paupière supérieure en plissant la peau $\mathrm{du}$ front, ou au contraire déplisser celleci. Il pourrait ainsi participer aux expressions de gaieté et d'étonnement $[3,12]$.

\section{architecture fonctionnelle labiale et péri-labiale}

(fig. 2)

Au-delà des caractéristiques générales et moyennes que nous venons d'évoquer, le sourire est une mimique délicate, hautement variable d'un individu à l'autre, et subtilement modulée chez un même sujet en fonction du sentiment qu'elle exprime (joie, tendresse, bienveillance, consentement, malice, mais aussi embarras, arrogance, mépris...). Cette dernière capacité, exclusivement humaine, repose sur une architecture musculo-fibreuse spécifique, résultant de l'interaction de trois structures labiales et péri-labiales : le SMAS (Système Musculo-Aponévrotique Superficiel), le sillon naso-labial et le modiolus.

Le concept de SMAS traduit l'importance fonctionnelle particulière que revêt le compartiment sous-cutané dans la région faciale : c'est un réseau tridimensionnel musculo-fibro-élastique organisé et continu, reliant les muscles faciaux au derme. Ghassemi a observé qu'il présente au niveau de la région labiale une structure spécifique qui ren- 


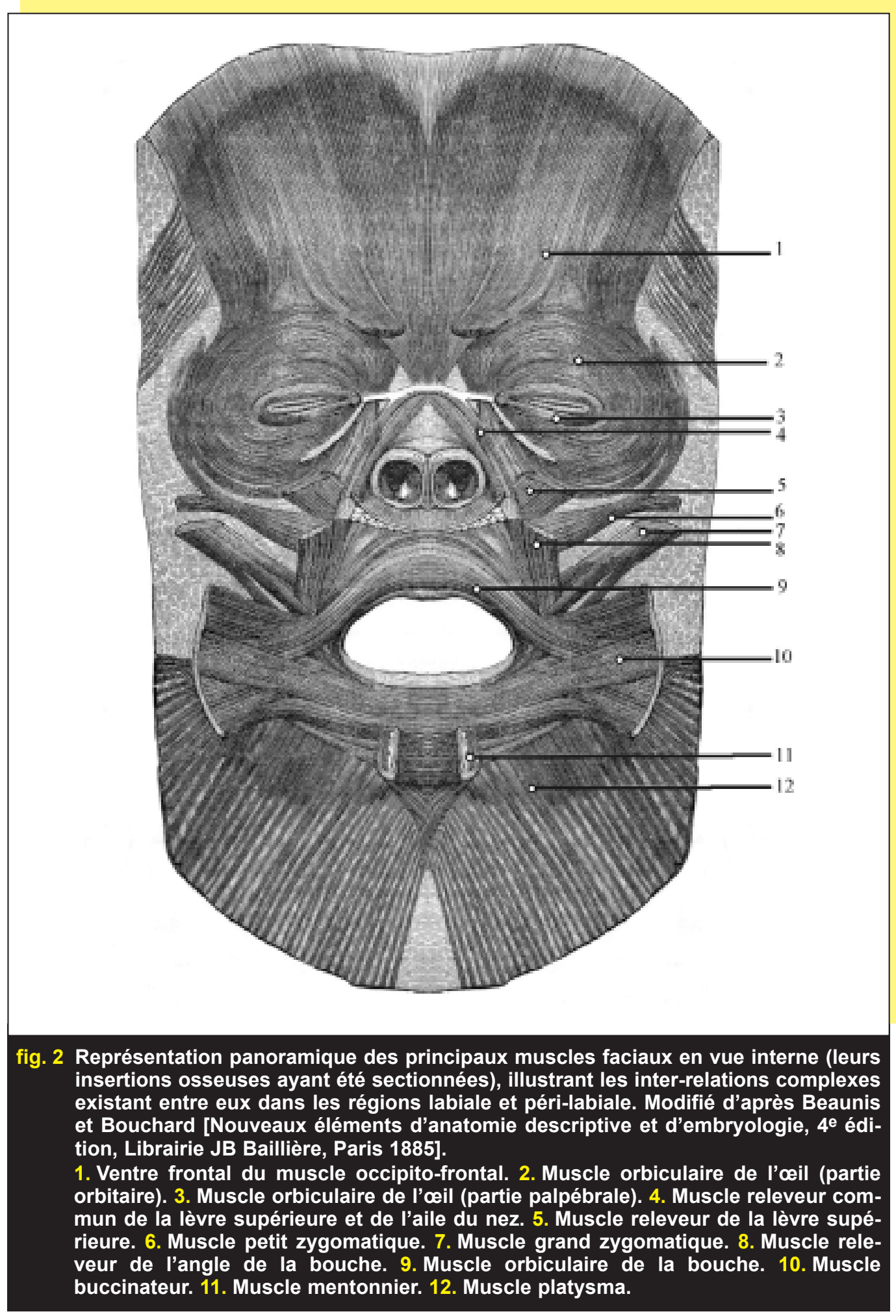


forcerait les rapports des muscles avec les téguments labiaux. La transition entre ce modèle architectural labial du SMAS, et celui rencontré dans les autres régions de la face s'effectue de manière brutale au niveau du sillon naso-labial[13]. Ce dernier, considéré par certains auteurs comme la simple résultante de la contraction des muscles élévateurs de la lèvre supérieure et de la commissure[14], est pour Rubin la structure-clé du sourire. Il serait en effet constitué de tissu fibreux, de fibres émanant des muscles releveur et releveur commun de la lèvre supérieure (certaines s'insérant dans le sillon, d'autres ne faisant que le traverser pour rejoindre leurs insertions basses), et de fibres musculaires propres qu'il désigne sous le terme de «muscle du sillon»[15]. Le sourire débuterait ainsi par une élévation de la lèvre supérieure en direction du sillon nasolabial, par l'action combinée des muscles élévateurs de la lèvre supérieure et $d u$ muscle du sillon. La résistance rencontrée à ce niveau entraînerait un recrutement musculaire plus important (notamment des muscles élévateurs de la commissure), se traduisant par un bombement de la pommette lié à la compression des par- ties molles jugales au-dessus du sillon. D'autres auteurs ont décrit cette diminution de la distance séparant la lèvre supérieure du sillon au cours du sourire[9].

Cette charpente musculo-fibreuse labiale est complétée par le modiolus, carrefour musculaire rétro-commissural dont l'organisation est centrée sur le muscle orbiculaire de la bouche, vers lequel convergent les muscles responsables des mouvements labiaux : muscles buccinateur, grand zygomatique, releveur et abaisseur de l'angle de la bouche, risorius, platysma et muscles incisifs[16]. Cette structure, dont la nature fibreuse est mise en doute par certains auteurs[2,17], constitue pour le muscle orbiculaire de la bouche, qui ne possède pas d'insertion osseuse directe, une aire d'insertion latérale dont la position peut être avantageusement modifiée par les autres muscles faciaux. L'orbiculaire des lèvres dispose ainsi de ce que l'on pourrait qualifier d'insertion distale mobile, pouvant changer de position plusieurs fois par minute, lui permettant d'assurer, en synergie avec les autres muscles faciaux, une large gamme de mouvements labiaux complexes[18].

\section{conclusion}

Les régions labiale et péri-labiale possèdent donc une architecture musculofibreuse tridimensionnelle spécialisée, leur permettant de transcrire la palette quasiment infinie, et personnelle, des sentiments humains, dont le sourire est une des manifestations les plus subtiles. 


\section{bibliographie}

1. Rouvière $\mathrm{H}$.

Anatomie humaine

descriptive,

topographique

et fonctionnelle

(Tome 1. Tête et Cou).

Masson et Cie Ed. Paris 1974.

2. Caix P

Anatomie

de la région labiale.

Ann Chir Plast Esthet 2002;47:332-345.

3. Brix M, Raphaël B La fonction labiale. Ann Chir Plast Esthet 2002:47:357-369.

4. Couly G

Anatomie microscopique et architecture musculaire de la commissure des lèvres

du nouveau-né

et de l'adulte.

Rev Stomatol 1976; 77(6):789-799.

5. Poirier P, Charpy $A$ Nicolas A.

Traité d'anatomie humaine.

Masson et Cie Ed. Paris 1912.

6. Pessa JE, Zadoo VP, Garza PA, Adrian EK Jr, Dewitt Al, Garza JR. Double or bifid zygomaticus major muscle :

anatomy, incidence and clinical correlation. Clin Anat 1998;11:310-313.
7. Duchenne (de Boulogne) G Mécanisme de la physionomie humaine.

Paris 1862.

8. Spiegel JH, DeRosa J.

The anatomical relationship between the orbicularis oculi muscle and the levator labii superioris and zygomaticus muscle complexes.

Plast Reconstr Surg 2005; 116(7):1937-1942.

9. Paletz JL, Manktelow RT, Chaban R.

The shape

of a normal smile: implications for facial paralysis reconstruction. Plast Reconstr Surg 1994; 93(4):784-789.

10. Rubin L.

The anatomy of smile: its importance

in the treatment of facial paralysis. Plast Reconstr Surg 1974; 53(4):384-387.

11. Ekman P, Davidson RJ, Friesen WV.

The Duchenne smile: emotional expression and brain physiology (II). J Pers Soc Psychol 1990;58(2):342-353.

12. Bolender CJ, Gay R. Anatomie du sourire. Rev Orthop Dento Faciale 1987;21:29-43.
13. Ghassemi $A$, Prescher $A$, Riediger D, Axer H. Anatomy of the SMAS revisited.

Aesth Plast Surg 2003; 27(4):258-264.

14. Patrinely JR, Anderson RL. Anatomy

of the orbicularis oculi and other facial muscles. Adv Neurol 1988;49: 15-23.

15. Rubin L.

The anatomy of the nasolabial fold: the keystone of the smiling mechanism.

Plast Reconstr Surg 1999: 103(2):687-691.

16. Lightoller GS.

Facial muscles.

J Anat 1925;60:1-85.

17. Greyling LM, Meiring JH. Morphological study on the convergence of the facial muscles at the angle of the mouth. Acta Anat (Basel) 1992; 143(2):127-129.

18. Pelissier P, Pistre $V$, Bustamante K, Martin D, Baudet J.

Le modiolus.

Anatomie comparée, rappels embryologique et physiologique, intérêt chirurgical. Ann Chir Plast Esthet 2000;45:41-47. 


\section{SUMMARY}

\section{Muscular anatomy of smile}

G. CLÈDES, R. FELIZARDO, P. CARPENTIER

Facial characteristics of smile lay on the action of facial muscles, organized in labial and orbitopalpebral bands. Those muscles, whom action is usually described individually, mesh with specific structures to produce combined cutaneous effects leading to the high intra- and inter-individual variability of facial expression. Those structures, SMAS (Superficial MusculoAponevrotic System), naso-labial fold and modiolus, give to the labial and peri-labial areas a specialized tri-dimensional musculo-aponevrotic architecture.

keywords: smile, muscular anatomy, lips, SMAS, naso-labial fold, modiolus. 